Research Paper

\title{
E2F8 regulates the proliferation and invasion through epithelial-mesenchymal transition in cervical cancer
}

\author{
Lee Kyung Kim,2, Sun-Ae Park², Kyung Jin Eoh ${ }^{1}$, Tae-Hwe Heo², Young Tae Kim ${ }^{1}{ }^{\varpi}$ and Hee Jung Kim² ${ }^{2}$ \\ 1. Institute of Women's Life Medical Science, Division of Gynecologic Oncology, Department of Obstetrics and Gynecology, Yonsei University College of \\ Medicine, Seoul, 03722, South Korea \\ 2. Laboratory of Pharmacoimmunology, Integrated Research Institute of Pharmaceutical Sciences, College of Pharmacy, The Catholic University of Korea, \\ Bucheon, 14662, South Korea
}

\begin{abstract}
$\triangle$ Corresponding authors: Young Tae Kim, MD, PhD, Institute of Women's Life Medical Science, Division of Gynecologic , Oncology, Department of Obstetrics and Gynecology, Yonsei University College of Medicine, Seoul, 03722, South Korea (e-mail: ytkchoi@yuhs.ac), Tel.:+82-2-2228-2814, Fax: +82-2-313-8357 and Hee jung Kim, PhD, Laboratory of Pharmacoimmunology, Integrated Research Institute of Pharmaceutical Sciences, College of Pharmacy, The Catholic University of Korea, Bucheon, 14662, South Korea (e-mail: hjk0114@hanmail.net), Tel.:+82-2-2164-4088, Fax:+82-2-2164-4059
\end{abstract}

(c) The author(s). This is an open access article distributed under the terms of the Creative Commons Attribution License (https://creativecommons.org/licenses/by/4.0/). See http://ivyspring.com/terms for full terms and conditions.

Received: 2019.06.18; Accepted: 2019.11.11; Published: 2020.01.01

\begin{abstract}
The transcription factor E2F is an important modulator of the cell cycle, and the unrestricted activation of E2F-dependent transcription is considered to be an important driver of tumor formation and progression. E2F8 is known to play an important role in embryonic development and cell cycle control by inhibiting E2F1. However, it is not yet known whether E2F8 is involved in the progression of cervical cancer. In this study, the functional consequences of E2F8 knockdown in vitro and in vivo were explored. To demonstrate the function of E2F8 in cell proliferation, migration and invasion, we knocked down E2F8 in cervical cancer cell lines; in vitro and in vivo experiments using this knockdown showed that E2F8 potently induced the expression of epithelial-mesenchymal transition (EMT) markers. Finally, clinical data confirmed that E2F8 was a significant predictive factor for progression-free survival, and that patients with cervical cancer who exhibited high expression of E2F8 showed high FIGO stages and frequent recurrence rates compared to patients with low E2F8 expression. In conclusion, our study suggests that E2F8 is highly correlated with the progression-free survival of cervical cancer patients.
\end{abstract}

Key words: E2F8, invasion, migration, cervical cancer, epithelial-mesenchymal transition

\section{Introduction}

As one of the most common malignant gynecological tumors, cervical cancer is responsible for 10 to $15 \%$ of all female cancer related deaths worldwide and is the second leading cause of death from female cancers, exceeded only by breast cancer $[1,2]$. Moreover, cervical cancer is associated with a high risk of morbidity and mortality due to metastasis and recurrence $[3,4]$. Therefore, biomarkers of early prognosis, metastasis and novel treatment targets are urgently needed to improve the survival of cervical cancer patients.

Recent studies have shown that the E2F family of transcription factors has many regulatory functions involved in cell proliferation, differentiation, DNA repair, cell cycle and apoptosis $[5,6]$. So far, the E2F family has eight members, named E2F1 to E2F8. Depending on structure and function, the E2F family can be divided into two groups (standard E2F1-6 and unstructured E2F7-8) and four subgroups (E2F1-3a, E2F3b-5, E2F6 and E2F7-8). There is also evidence that $\mathrm{E} 2 \mathrm{~F} 7 / 8$ is involved in the transcriptional suppression of the cell cycle gene for seven or eight years of DNA damage $[7,8]$. Although the exact role of E2F7 / 8 in DNA damage is not clear, transcription in cell cycle processes that enable optimal DNA recovery is one possibility because high levels of E2F7 / 8 were shown to inhibit proliferation within the laboratory [9]. Particularly, recent literature reported that expression of E2F8 was markedly enhanced in multiple carcinomas, which implies that it is involved 
in oncogenesis and cancer progression [10]. However, the biological role and clinical significance of E2F8 in the progression of cervical cancer has not yet been identified in relation to the epithelial-mesenchymal transition (EMT).

In this study, we investigated the functional role of E2F8 in the progression of cervical cancer. Our results show that E2F8 strengthened the growth, migration, and invasion of cervical cancer cells through an EMT signaling pathway. Our study suggests that E2F8 is a promising prognostic factor and target for cervical cancer treatments.

\section{Materials and Methods}

\section{Patients and tissue samples}

In total, 80 women who underwent surgery between 2012 and 2018 at Yonsei Severance Hospital, Yonsei University, seoul, korea, were included in this study. This study was conducted according to the principles of the Helsinki Declaration and ethical guidelines of the Yonsei Severance Hospital Ethics Committee. A sample of newly diagnosed stage IA to IVB cervical cancer (international gynecology and gynecology) was evaluated without prior diagnosis. Additionally, normal cervical tissues from 20 patients undergoing simple hysterectomies because of uterine leiomyomata were obtained as controls. The study was approved by the Yonsei Severance Hospital Ethics Committee and approved by all patients. All samples were immediately frozen in liquid nitrogen until RNA extraction and stored at $-80^{\circ} \mathrm{C}$.

\section{Cell lines}

Human epithelioid cervical adenocarcinoma (HeLa) cells were obtained from the Korean Cell Line Bank (Seoul, Korea). The epidermoid cervical cancer carcinoma line, established from transfer in small intestine (Caski cells) and ME180 cells, was obtained from the American Type Culture Collection (ATCC, Rockville, MD, USA). Human keratinocyte HaCaT cells were obtained from the Creative Bioarray (Shirley, NY, USA).

HeLa cells were cultivated in Dulbecco's modified Eagle's medium (WelGENE Inc, Korea) and Caski, ME180 and HaCaT cells were cultivated in the RPMI-1640 media (WelGENE Inc., Korea). The culture medium contained $10 \%$ (vol/vol) fetal bovine serum and penicillin/streptomycin. The cell lines were maintained at $37^{\circ} \mathrm{C}$ in humidified air with $5 \% \mathrm{CO}_{2}$ and $95 \%$ air. The passage number of cells was $<20$ in all experiments.

\section{Quantitative real-time PCR (qRT-PCR)}

RNA was extracted from cancer tissues or cultured cells using TRIzol reagent (Bioline, London,
UK). In accordance with the manufacturer's instructions, the total RNA was reversed to cDNA using the reverser reagent kit (Bioline). Real-time PCR analyses were conducted employing a SYBR Green Real-Time PCR Kit (Bioline). Settings for the amplification of E2F8 were as follows: initial denaturation at $95^{\circ} \mathrm{C}$ for 3 minutes, 40 cycles with denaturation at $95^{\circ} \mathrm{C}$ for 15 seconds, annealing at $60^{\circ} \mathrm{C}$ for 60 seconds, and elongation at $72^{\circ} \mathrm{C}$ for 60 seconds, then final elongation at $72^{\circ} \mathrm{C}$ for 5 minutes. qRT-PCR was accomplished using an ABI StepOnePlus Real-Time PCR System (Applied Biosystems, Foster City, CA, USA). The results were normalized with the expression of U6. The relative change in the expression of mRNA was calculated by the $2-\Delta \Delta C T$ method. All qRT-PCR experiments were replicated at least three times.

\section{Small interfering RNA (siRNA) transfection}

E2F8 siRNA (siE2F8) and negative control siRNA (siNC) were obtained from Genolution (Genolution Pharmaceuticals Inc., Seoul, Korea). Seeded cells $\left(5 \times 10^{4}\right.$ cells/well $)$ into 6-well plates, using the G-fectin Kit (Genolution Pharmaceuticals) in accordance with the manufacturer's instruction for transfection with $30 \mathrm{nM}$ siRNA in phosphatebuffer saline (PBS). The siRNA-transfected cells were used in in vitro assays 48 hours after transfection. The experiments were repeated at least three times.

\section{E2F8 gene silencing by shRNA}

Target shRNA sequences against human E2F8 were designed based on the sequence available from the Gene Bank. The sequence of the E2F8 shRNA was TRCN0000017428: 5'-GCCGCAAAGACAAGTCTTT AA-3'. Oligonucleotides were formed by annealing, then the constructs were cloned into the pLKO.1 vector. HEK293T cells were transfected with shRNA-coded lentiviral vector DNA and packaging vectors (pCMV-VSVG, psPAX2, and pMC2.G) using Lipofectamine 2000 (Life Technologies, Carlsbad, CA, USA). The viral medium was collected at both 48 and 72 hours post-transfection. Cells were infected in the viral medium along with $8 \mu \mathrm{g} / \mathrm{ml}$ polybrene, followed by selection with puromycin.

\section{Cell proliferation assay}

The Cell Counting Kit-8 (CCK-8, Dojindo, Japan) assay was used to assess cell proliferation. Cells $(5 \mathrm{x}$ $10^{4}$ cells/well) were seeded into 6-well flat-bottomed plates in $2 \mathrm{~mL}$ of complete medium. After incubation of cells overnight to allow for cell attachment and recovery, the cells were transfected with siNC or siE2F8 for 24, 48, 72 or 96 hours. An aliquot of $200 \mu \mathrm{L}$ of the CCK-8 solution was added to each well and 
incubated for 1 hour at $37^{\circ} \mathrm{C}$. The optical density (OD) was measured at $450 \mathrm{~nm}$ using an auto-microplate reader to calculate the number of viable cells in each well. The assay was performed in triplicate.

\section{Colony formation assay}

ME180 cells were transfected with shE2F8 or scrambled $\left(5 \times 10^{4}\right)$ suspensions and then incubated in an upper layer of $1 \%$ agar noble (A5431 Sigma-Aldrich, St. Louis, MO, USA) in 2x RPMI with $10 \%$ fetal bovine serum. The suspension was overlaid on $0.6 \%$ basal agar with $10 \%$ fetal bovine serum in a 6-well plate and placed at room temperature until the agarose solidified. The plate was transferred to a 5\% $\mathrm{CO}_{2}$ incubator, incubated at $37^{\circ} \mathrm{C}$ for 3 weeks, and stained with crystal violet. Colonies with a diameter greater than $0.5 \mathrm{~mm}$ were counted using the ImageJ software (NIH Bethesda, MD, USA).

\section{Wound healing migration assay}

Cell migration was evaluated with a wound healing assay. About $5 \times 10^{5}$ cells were seeded into 6-well plates with serum-containing medium and allowed to grow to $90 \%$ confluency in complete medium. The serum-containing medium was eliminated and the cells were serum starved for $24 \mathrm{~h}$. When the cell density reached $100 \%$, artificial homogenous wounds were created by scratching a single layer using a sterile $200 \mu \mathrm{L}$ pipette tip. After scratching, the cells were washed with phosphate buffer saline (PBS). Using the microscope, cell migration into the wound were captured for 0,24 , and $48 \mathrm{~h}$. The width of the scratch was measured using the NIH ImageJ software and calculated as the percentage of the closed scratch area (width at $0 \mathrm{~h} /$ width $48 \mathrm{~h}$ ). The results were standardized to control cells. The migrated cells were counted in 10 fields under an 20x objective lens. Original magnification as 200x. The experiments were performed in triplicate.

\section{Transwell migration assay}

The migration assay was designed using $6.5 \mathrm{~mm}$ diameter Transwell plates (Corning Costar, Cambridge, MA, USA) with $8 \mu \mathrm{m}$ pore filters. Cells (5 $x 10^{5}$ ) were seeded in the upper chamber in serum-free medium and complete medium was added to the lower chamber. Cells were incubated for 48 hours in the migration chamber set to $37^{\circ} \mathrm{C}$ and $5 \%$ $\mathrm{CO}_{2}$. Non-invasive cells were removed from the upper chamber with a cotton swab. The cells that intruded into the lower surface of the filter were dyed (Diff Quik, Sysmes, Kobe, Japan) and counted with a hemocytometer. This assay was repeated at least three times.

\section{Matrigel invasion assay}

The Matrigel invasion assay was performed using BD Biocoat Matrigel Invasion Chambers (pore size: $8 \mathrm{~mm}$, 24-well; BD Biosciences, Bedford, MA, USA) according to the manufacturer's protocol. Cells $\left(5 \times 10^{5}\right)$ were seeded in the upper chamber in serum-free medium and complete medium was added to the lower chamber. Cells were incubated for $48 \mathrm{~h}$ in the Matrigel invasion chamber set to $37^{\circ} \mathrm{C}$ and $5 \% \mathrm{CO}_{2}$. Non-invasive cells were removed from the upper chamber with a cotton swab. The cells that entered the lower surface of the filter were stained (Diff Quik, Sysmes, Kobe, Japan) and counted with a hemocytometer. This assay was repeated at least three times.

\section{Western blot analysis}

RIPA buffer (Thermo Fisher Scientific Inc. Waltham, MA, USA) was used to extract proteins. We measured the protein concentrations using a Pierce BCA Protein assay kit (Thermo Fisher Scientific). The protein was boiled with a $5 x$ sample buffer to dissolve it further in a $10 \%$ SDS-polyacrylamide gel, followed by electrophoretic transfer to a polyvinylidene difluoride membrane (Milipore, Billica, MA, USA). After blocking with $5 \%$ fat-free dried milk in $1 \mathrm{x}$ Tris-buffered saline containing $0.1 \%$ tween 20 (pH 7.6) at room temperature for 1 hour, the membrane was continuously stirred and incubated with primary antibody at $4^{\circ} \mathrm{C}$ overnight.

\section{Xenografts in mice}

BALB/c mice ( $n=30,5-6$ weeks of age, Orient Bio, Seongnam, Korea) were kept in aseptic conditions under constant temperature and humidity (Yonsei Medical University protocol). Each mouse received a subcutaneous dorsal scapula injection of a $200 \mu \mathrm{L}$ suspension of HeLa cells pretreated with siE2F8 and siNC for $48 \mathrm{~h}\left(1.0 \times 10^{6}\right.$ cells/flank, xenograft $\left.\mathrm{n}=18\right)$. ME180 cells pretreated with shE2F8 or shscrambled control were transplanted $\left(2.0 \times 10^{6}\right.$ cells/flank, xenograft $n=12$ ) into the subcutaneous dorsal scapula injection.

Calipers were used to measure the tumor sizes twice a week. The tumor volume was calculated using a simplified equation to estimate a rotational ellipsoid (length $\mathrm{x}$ width ${ }^{2} \times 0.5$ ). Each tumor was harvested 40 days post-treatment.

\section{Magnetic resonance (MR) imaging in mice}

MR images were obtained using Brucker Biospec 94/24 USR (9.4T) small animal scanner (35 mm diameter cage coil, Brut BioSpin MRI, Ettlingen, Germany). A custom-built cradle was used to immobilize each mouse during the MR imaging 
process. At the beginning of each imaging session, the T2-weighted image was obtained using the quick acquisition setting. These images were used to confirm that the animal was in the correct position inside the magnetic bore. A $1.5 \%$ isoflurane and $\mathrm{O}_{2} / \mathrm{N}_{2} \mathrm{O}(1: 1)$ mixture at a $0.7 \mathrm{~L} / \mathrm{min}$ flow rate was used as anesthesia during the MR imaging. Breathing was monitored using an air pillow. The body temperature of the mice was maintained within acceptable limits using circulating warm water.

\section{Hematoxylin and eosin (H\&E) staining}

Mice were euthanized after the MRI. Tumor tissue was collected, fixed with $4 \%$ paraformaldehyde for $24 \mathrm{~h}$, washed in PBS, and then embedded in paraffin. Two-micrometer sections were stained with hematoxylin and eosin following standard procedures.

\section{Statistical analyses}

IBM SPSS version 24 for Windows (SPSS Inc., Chicago, IL, USA) was used for statistical analyses. We used Pearson's $\chi^{2}$ tests, Student's $t$-tests, and Fisher's exact tests to evaluate the associations between E2F8 expression and clinicopathological characteristics. In order to evaluate the performance of the model with respect to their discriminating capability, the chi-square value of the log rank test was used in the receiver operating characteristic (ROC) analysis. The median value (1.2962) was set to
(A)

E2F8

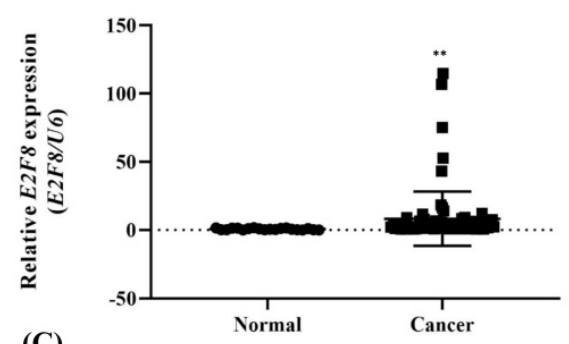

(C)

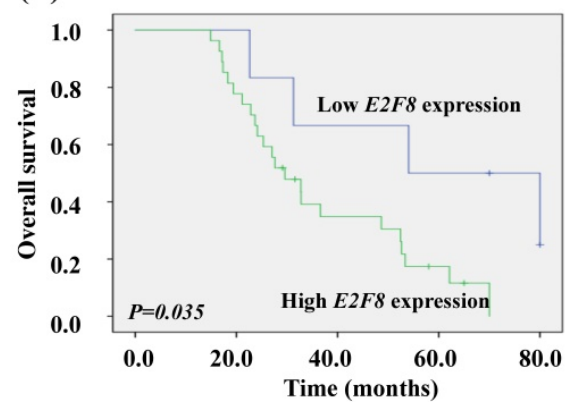

(B)

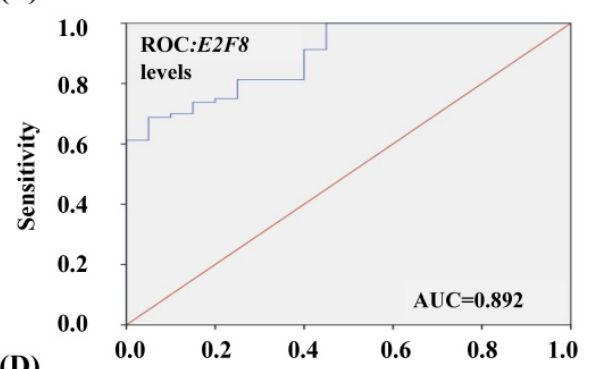

(D)

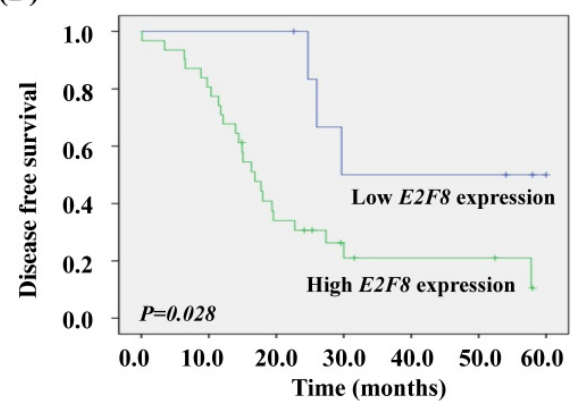

Figure 1. The clinical significance of E2F8 expression in cervical cancer tissue. (A) The E2F8 expression was significantly higher in cervical cancer tissues $(n=80)$ than in non-cancerous tissues $(n=20)$. E2F8 expression was determined using qRT-PCR and is expressed relative to the control value. Data are expressed as mean $\pm S D$. ${ }^{* *} p<0.01$ vs. non-tumor control. (B) ROC curve for prognostic predictions for patients by E2F8 levels. The AUC is shown in the plots. ROC, receiver operating characteristic; AUC, area under the curve. (C) Kaplan-Meier curves for the overall survival and (D) disease-free survival of cervical cancer patients with different expression levels of E2F8. the cutoff value. These groups were classified as high and low E2F8 expression groups, respectively, at the above and below values of the cut-off value of 1.2962 . The entire survival time was analyzed using the Kaplan-Meier method. The log rank test was used to estimate the differences between groups. The stepwise Cox proportional hazards model was used for multivariate survival analysis of the parameters which were significant in the univariate analysis. The statistical tests were two-sided and p-values of less than 0.05 were considered significant.

\section{Results}

\section{E2F8 expression was elevated in cervical cancer and correlated with poor prognosis}

To determine whether E2F8 expression in tissues was associated with the clinicopathologic characteristics of cervical cancer, we evaluated the expression of E2F8 in cervical cancer tissue $(n=80)$ and corresponding normal tissue $(\mathrm{n}=20)$. The E2F8 expression in cervical cancer tissues was more than 11.07 times that of non-cancerous tissues $(p=0.001$; Figure 1A).

The risk model for E2F8 data showed a predicted AUC of $0.892(p=0.00000007$; Figure 1B.). We compared the characteristics of patients with high E2F8 expression $(n=62)$ to those with low E2F8 expression ( $\mathrm{n}=18$; Table S1). Kaplan-Meier survival analysis demonstrated that cervical cancer patients with low E2F8 levels exhibited longer overall survival and disease-free survival than cervical cancer patients with high E2F8 levels $(p=0.035$ and 0.028, respectively Figure $1 C$ and D).

Moreover, according to univariate and multivariate analyses using the Cox proportional hazards model, E2F8 expression was a significant predictive factor for stage and recurrence (stage, univariate hazard ratio $(\mathrm{HR})=$ 1.844 (1.276-2.663), $\quad p=0.001$, multivariate $\mathrm{HR}=1.816 \quad(1.258$ 2.622), $p=0.001$; recurrence, univariate $H R=7.545$ (3.535-16.103), $p=0.000000017$, multivariate $\mathrm{HR}=$ 7.823 (3.605-16.973), $p=0.0000001$; Table 1). Both univariate and multivariate proportional hazard analyzes showed that stage and recurrence were independent prognostic factors for overall survival. 
Table 1. Univariate and multivariate analysis of various factors for overall survival

\begin{tabular}{|c|c|c|c|c|}
\hline & \multicolumn{4}{|l|}{ OS } \\
\hline & \multicolumn{2}{|l|}{ Univariate analysis } & \multicolumn{2}{|l|}{ Multivariate analysis } \\
\hline & HR (95\% CI) & $\mathbf{P}$ & HR (95\% CI) & $\mathbf{P}$ \\
\hline E2F8 expression & $1.108(0.481-2.556)$ & 0.809 & & \\
\hline Age, years (continuous) & $1.003(0.974-1.033)$ & 0.837 & & \\
\hline FIGO stage & $1.844(1.276-2.663)$ & 0.001 & $1.816(1.258-2.622)$ & 0.001 \\
\hline cell type & $1.026(0.720-1.461)$ & 0.889 & & \\
\hline Lymph node metastasis & $1.939(0.977-3.847)$ & 0.054 & & \\
\hline Lymphovascular invasion & $1.385(0.658-2.915)$ & 0.39 & & \\
\hline Recurrence & $7.545(3.535-16.103)$ & 0.000000017 & $7.823(3.605-16.973)$ & 0.000000195 \\
\hline tumor size & $1.365(0.849-2.195)$ & 0.199 & & \\
\hline
\end{tabular}

OS, overall survival; HR, hazard ratio; FIGO, The International Federation of Gynecology and Obstetrics

\section{E2F8 expression was elevated in cervical cancer cell lines and correlated with cell proliferation}

To investigate the E2F8 expression in cervical cancer cells, several cell lines were examined for mRNA and protein levels of E2F8 (Figure 2A and B).
(A)

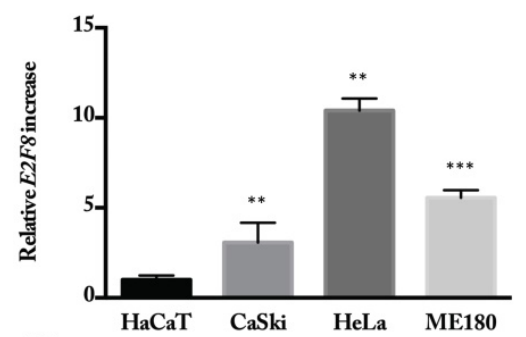

(C)

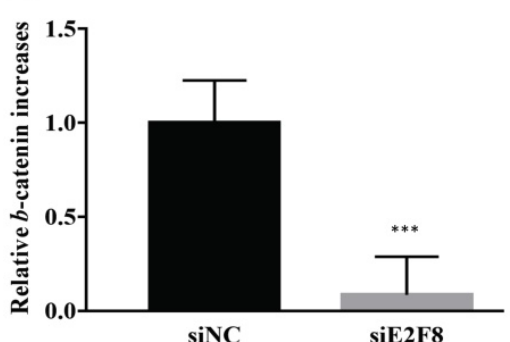

(E)

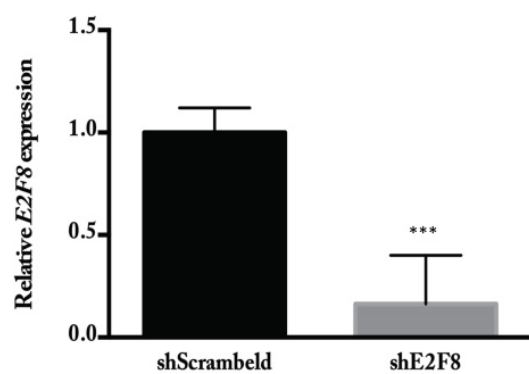

(B)

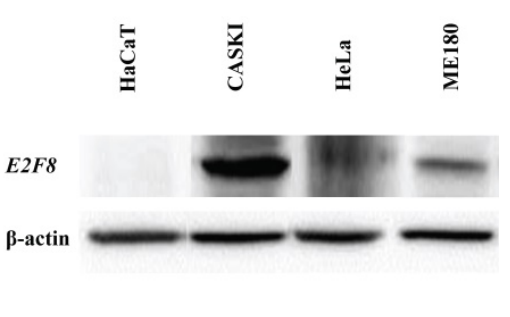

(D)

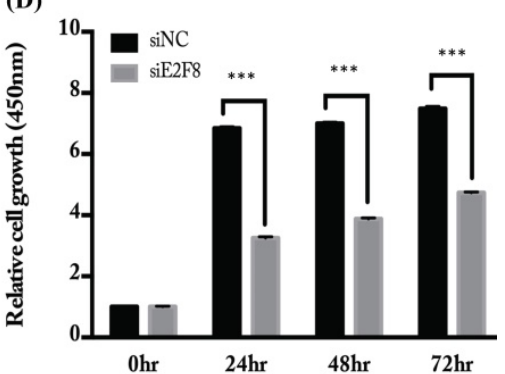

(F)

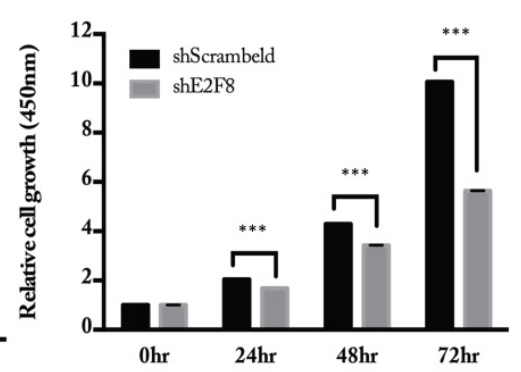

Figure 2. E2F8 expression in cervical cancer cell lines and correlation with suppressed cell proliferation. (A, B) The expression levels of E2F8 RNA and protein were significantly higher in some cervical cancer cell lines (CaSki, HeLa, and ME180) compared to that in normal control cells. (C) Using siE2F8, E2F8 knockdown was performed in E2F8-elevated HeLa cell lines. (D) The proliferation of HeLa cells transfected with siE2F8 and negative control siNC was determined using the CCK-8 assay. (E) Using shE2F8, E2F8 knockdown was performed in E2F8-elevated ME180 cell lines. (F) The proliferation of ME180 cells transfected with shE2F8 and negative scrambled control was determined using the CCK-8 assay. Each assay was performed in triplicate. Data are means \pm standard deviation. $* * P<0.01, * * * P<0.001$ vs siNC and scrambled control.
As shown in Figure 2, CaSki, HeLa, and ME180 cells expressed higher levels of E2F8 than the control in cervical cancer cells. By transfecting siE2F8 and shE2F8, E2F8 was knocked down in HeLa and ME180 cells. The siE2F8 and shE2F8-transfected HeLa and ME180 cells also showed significant inhibition of cell proliferation (Figure 2C and E). E2F8 inhibited cell proliferation by $50 \%$ and $60 \%$ at $72 \mathrm{~h}$ post-transfection in HeLa and ME180 cell lines, respectively, relative to control cells (Figure 2D and F). Thereafter, colony formation assays confirmed that E2F8 inhibited cell colony formation and by $90 \%$ in ME180 cell lines relative to control cells (Figure 2G and F).

\section{E2F8 knockdown inhibited the migration and invasion of cervical cancer cells}

We investigated whether E2F8 affects the invasion and migration of cervical cancer cells. A Matrigel invasion assay was used to assess invasion after $48 \mathrm{~h}$. E2F8 knockdown in cervical cancer cell lines (HeLa and ME180) showed significant decrease in wound-healing. Therefore, the same cell lines were used to assess migration in si- and sh-E2F8 transfected cervical cancer cells (Figure 3A and $\mathrm{C}$ ). In addition, the invasiveness of these cells was compared to that of control cells. The E2F8 knockdown cells exhibited reduced invasiveness (Figure $3 \mathrm{~B}$ and $\mathrm{D}$ ). While uncontrolled cell proliferation is a common biological feature of all tumors, the key pathophysiological feature of malignant tumors in pathological conditions is the ability to penetrate the natural tissue barrier. Our findings suggest that E2F8 expression correlated with cancer invasion and metastasis. 
(A)

Oh

$12 \mathrm{~h}$

24h

36h

$48 \mathrm{~h}$

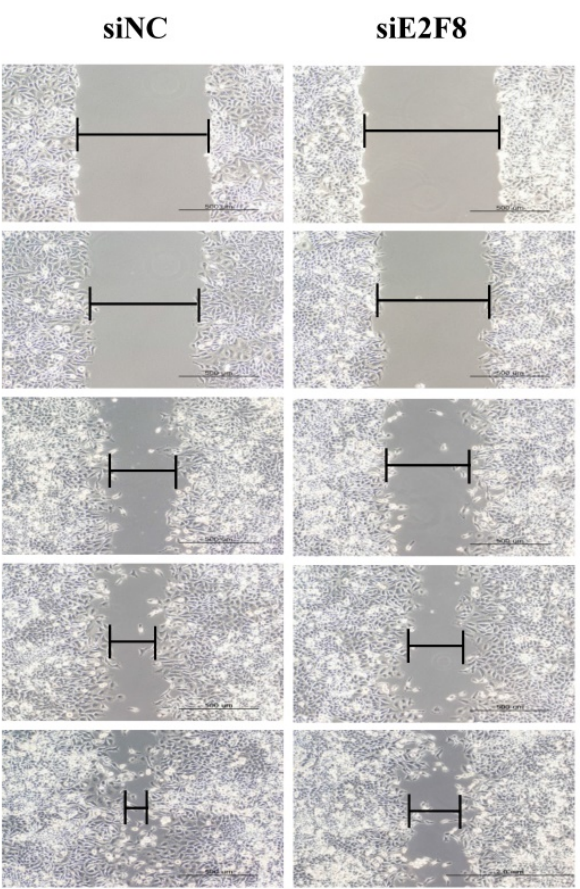

(B)
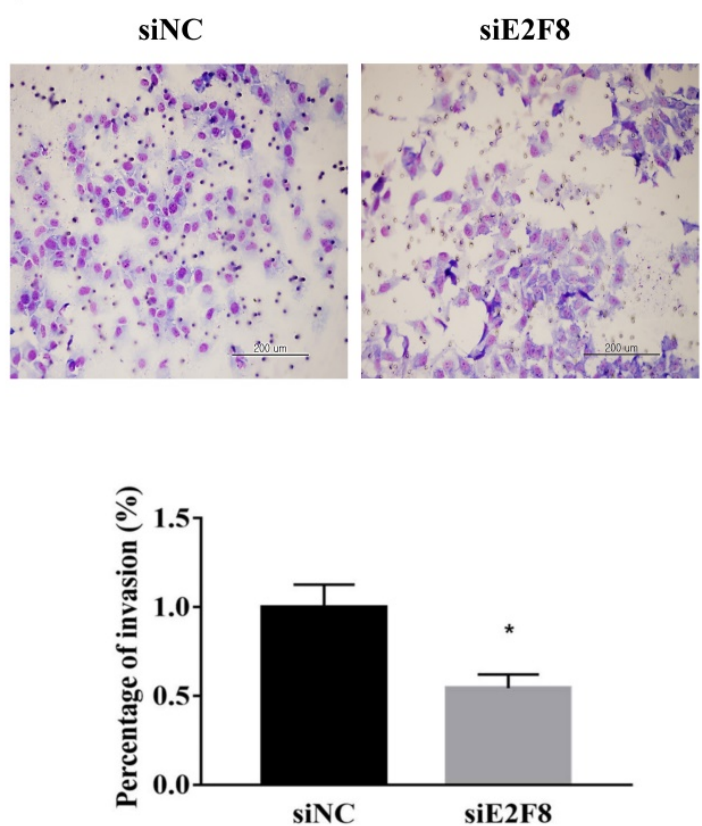

(C)
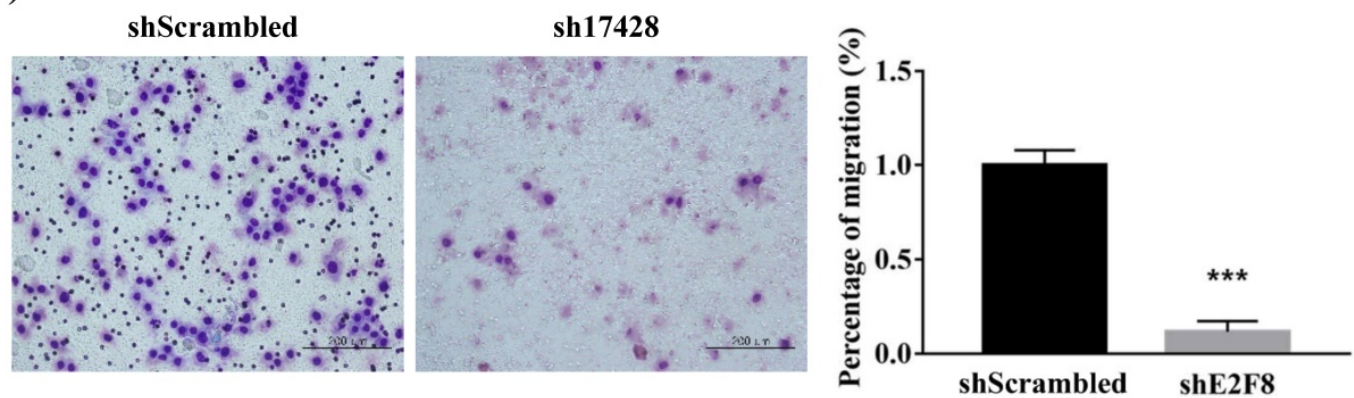

(D)
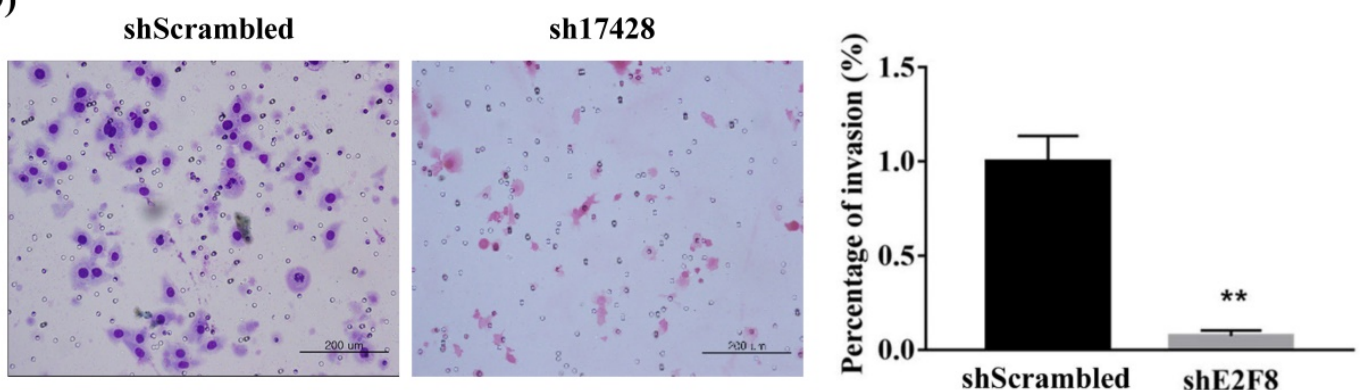

Figure 3. E2F8 promotes cell migration and invasion. (A, C) Wound healing assay observed under the optical microscope was used to determine cell migration using si and shE2F8. E2F8 knockdown was performed in E2F8-high HeLa and ME180 cell lines. (B, D) Cell invasion was observed under the optical microscope. Matrigel invasion assays were used to determine invasion after $48 \mathrm{~h}$ in HeLa and ME180 cells. Each assay was performed in triplicate. Data represent means \pm standard deviation. $* * P<0.01, * * * P<0.001$ vs. siNC and scrambled control.

\section{E2F8 knockdown reversed EMT signaling pathway-related genes in cervical cancer cells}

Because EMT is crucial in cell migration and invasion, the identification of factors associated with EMT can have a clinical impact $[11,12]$. Thus, we examined whether E2F8 was associated with EMT. Towards this, EMT-related markers were evaluated using real-time RT-PCR (Figure S1A and 4A) and
Western blotting (Figure S1B and 4B) after the si- and sh-RNA-mediated knockdown of E2F8 in HeLa and ME180 cells. Knockdown of E2F8 increased E-cadherin expression and decreased the expression of N-cadherin, b-catenin, vimentin, Wnt5, and Twist. These results indicate that the dysregulation of EMT-related genes may explain the involvement of E2F8 in cervical cancer cell migration and invasion. 


\section{E2F8 knockdown blocked tumor growth in a xenograft nude mouse model}

To explore whether E2F8 affected tumor growth in vivo, we implanted HeLa and ME180 cells, in which E2F8 was knocked down, as xenografts into nude mice (Figure S2A and Figure 5A). Tumor volume (Figure S2B and Figure 5B) and weights (Figure S2C and Figure $5 \mathrm{C}$ ) were measured. The mean tumor

(A)
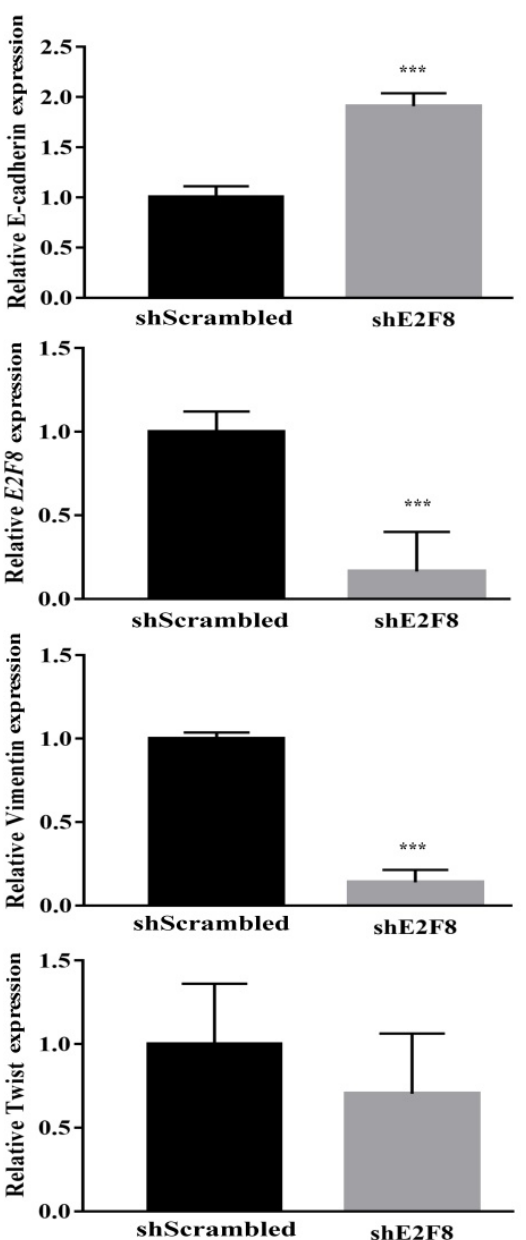

(B)

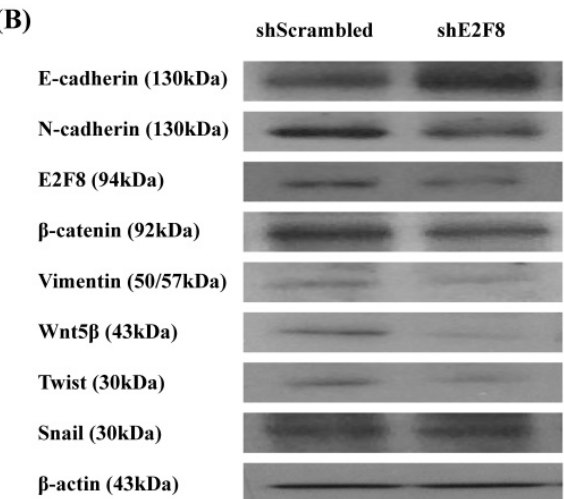

Figure 4. Effect of E2F8 Knockdown on EMT progression. (A) ME180 cells were transfected with shE2F8 or scrambled control. Real-time RT-PCR of EMT-related transcription factors after E2F8 knockdown in ME180 cells. Data represent means \pm SD. $* * P<0.01$, $* * * P<0.001$ vs. scrambled control. (B) Protein lysates were prepared using shE2F8- and scrambled control-transfected ME180 cells. volumes and weights of mice, which were implanted with siE2F8 transfected HeLa cells and shE2F8 transfected ME180 cells were significantly smaller than those of mice that had been impalnted with siand sh-scrambled control cells $(p<0.05$. Histological examination revealed that more cells with small nucleoli and irregular nuclear membranes were present in the E2F8 knockdown xenografts than that in the control xenografts (Figure S2D and Figure 5D). We further evaluated tumor size and activity using magnetic resonance imaging (MRI) (Figure S2E and Figure 5E). These findings suggested that E2F8 promoted tumor growth in vivo, and further supported our hypothesis that E2F8 is involved in the malignant transformation of cervical cancer cells.

\section{Discussion}

The deregulation of E2Fdependent transcription occurs frequently in many cancers and is considered an important factor in the uncontrolled proliferation of cancer cells. However, the function and contribution of E2F family members in various types of cancers is complex and poorly understood [13]. Most studies have indicated that $\mathrm{E} 2 \mathrm{~F}$ deregulation in tumors is a result of overexpression of proliferation-promoting transcription factors, rather than deregulation of the mainly inhibitory transcription factors. In non-small cell lung cancer and esophageal squamous cell carcinoma, tumor progression and poor outcomes correlate with overexpression of the proliferation-promoting transcription factor E2F1 [14, 15]. In contrast, tumor growth is enhanced by the downregulation of inhibitory E2F transcription factors such as E2F4 and E2F7, and such an imbalance may cause a switch from cell quiescence to growth promotion [16]. E2F protein is a proven modulatory factor in malignant progression in various cancers. The newly identified E2F8 has been reported to be an important proliferation factor in some human cancers [17]. A deeper understanding of the molecular mechanisms that determine the progression and metastasis of cervical cancer is essential for the 
development of more effective therapies and for the identification of new diagnostic indicators of cervical cancer.

Recent studies have shown that some abnormal molecular changes can play a central role in the development of cervical cancer [18]. However, the clinical significance and biological function of E2F8 in gynecological cancer, especially cervical cancer, remains unknown. Accordingly, in this study, the molecular function and clinical significance of E2F8 expression was investigated in cervical cancer cell lines. We found that E2F8 expression was higher in cervical cancer tissues than that in non-cancerous tissues. Moreover, E2F8 knockdown altered the cell growth, migration, and invasion of cervical cancer cells. The metastatic effects of E2F8 appeared to be mediated, at least in part, by regulation of the genes involved in cell migration, invasion, and EMT.
(A)

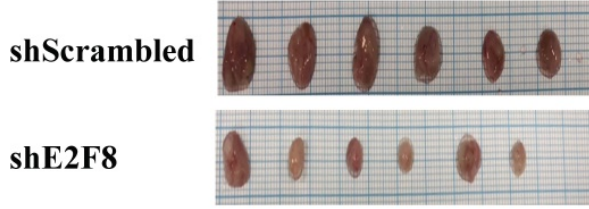

(C)

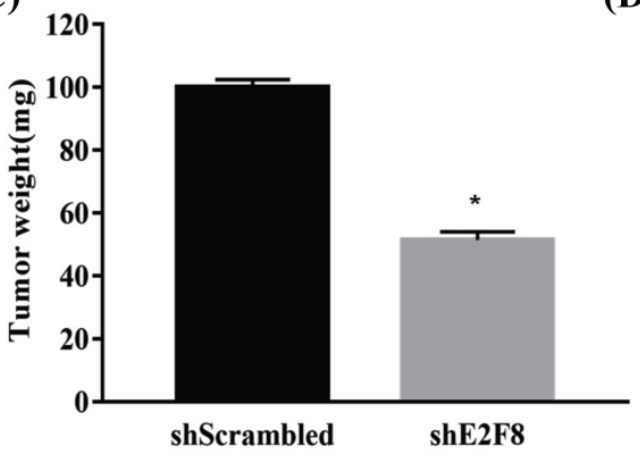

(B)
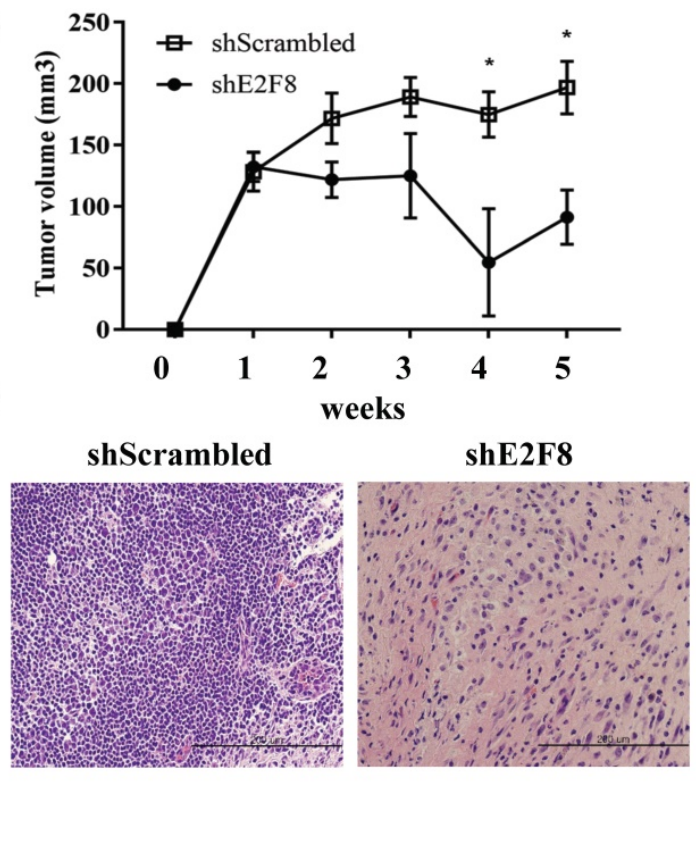

(E)

shScrambled
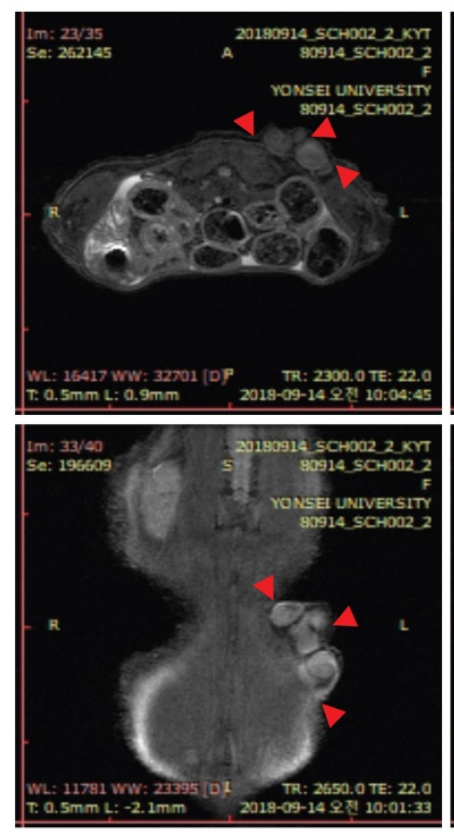

$\operatorname{shE2F8~}$
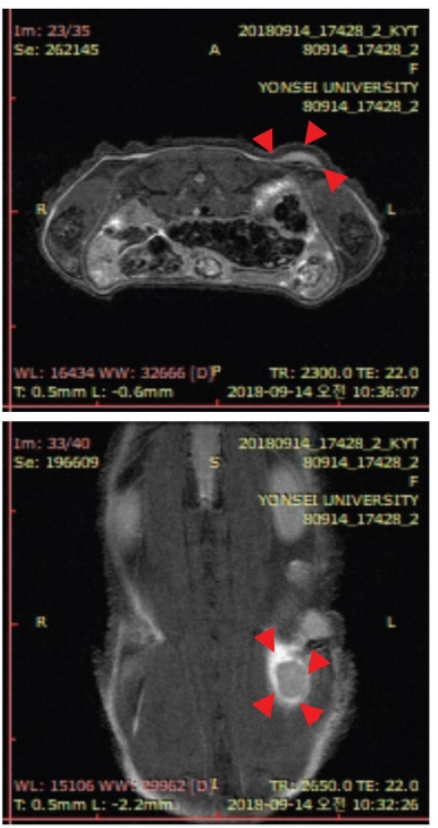

Figure 5. E2F8 knockdown decreases tumor size in a xenograft nude mouse model. (A) ME180 cells transfected with shE2F8 or scrambled control were injected subcutaneously into the right dorsal scapula area of nude mice. Gross images of tumor masses from representative mice from each group. (B) Tumor sizes in the experimental groups. (C) Tumor weights were compared after tumor harvest. (D) Hematoxylin and eosin (H\&E) staining of shE2F8-transfected ME180 cells (x200). (E) MRI image. *p<0.05 vs. scrambled control. 
There is some evidence that E2F8 might also be associated with human cancers. An increase in the E2F8 gene copy number was detected in melanoma [19] and increased expression was reported in ovarian cancer and hepatocellular carcinoma [20]. E2F8 knockdown caused an almost complete block of cell proliferation and a substantial induction of apoptosis, but did not inhibit normal bronchial epithelial cell growth. These results indicate that while E2F8 does not serve a role in normal cells, it plays an integral role as a tumor promoter in cancer cells.

To better understand the direct role of E2F8 in carcinogenesis, we targeted EMT signaling by knockdown of E2F8. The EMT involves alterations in cell phenotypes and several transcription factors have been implicated in the regulation of EMT-related gene expression. Although several studies have focused on transcriptional regulators in pathological EMT, few studies have evaluated the roles of transcription factors in cervical cancer [21]. The present study explored the molecular function of E2F8 expression in cervical cancer cell lines. Our findings showed that downregulated E2F8 expression was correlated with decreased cell growth, migration, and invasion of cervical cancer cells. This effect of E2F8 on tumor progression may be mediated by genes involved in cell migration, invasion, and the EMT signaling pathway, as well as genes that encode E-cadherin, $\mathrm{N}$-cadherin, $\beta$-catenin, vimentin, and Snail. Our findings suggested that E2F8 could potentially represent a novel biomarker and therapeutic target for cervical cancer. The loss of E-cadherin is thought to be an important event in the EMT, but N-cadherin reduces the intercellular connection between two adjacent endothelial cells, causing the cancer cells to slip [22]. In addition, $\beta$-catenin is more slowly mobile and weakens the related mesenchymal phenotype. The improvement in the expression of transcription factors, such as in snail and twist, is related to the loss of adhesion between cells [23]. Vimentin is the main component of the cytoskeleton of mesenchymal cells and its upregulation is induced by the EMT [24, 25].

We hypothesized that E2F8 may act as an important regulator for several signaling mechanisms related to the EMT. Our results suggest that E2F8 can contribute to the growth, invasion, and recurrence of cervical cancer through induction of the EMT. The recurrence rate of advanced cervical cancer after radical surgery is 15 to $30 \%$ and the prognosis of patients with recurrence is poor [26]. In order to improve the prognosis of cervical cancer patients, a firm prediction of relapse and progression is needed. The EMT signaling pathway contributes to tumor progression characteristics, including invasion, metastasis, and angiogenesis [27]. Furthermore, clinical outcome data from 80 patients with epithelial cervix cancer support the findings of our in vitro experiments.

In summary, our research suggests that E2F8 is correlated with the progression of cervical cancer via EMT signaling pathways. These results support E2F8 as a promising prognostic marker and therapeutic target for cervical cancer.

\section{Abbreviations}

E2F8: the E2 factor family 8; EMT: epithelialmesenchymal transition; CCK-8: cell counting kit-8.

\section{Supplementary Material}

Supplementary figures and tables. http://www.ijbs.com/v16p0320s1.pdf

\section{Acknowledgment}

This research was supported by the Research Fund 2019 of the Catholic University of Korea, and BK21 PLUS project of the NRF funded by the Ministry of Education of Korea (22A20130012250) and the Korea Health Technology R\&D Project through the Korea Health Industry Development Institute, funded by the Ministry of Health and Welfare, Republic of Korea (grant no. HI17C0321) and by the Basic Science Research Program through the National Research Foundation of Korea funded by the Ministry of Education, Science and Technology (grant no. NRF2018R1D1A1B07049578， NRF-2018R1D1A1B0749780 and 2018R1A6A1A03025108).

\section{Ethics approval and consent to participate}

The study was approved by the Yonsei Severance Hospital Ethics Committee and approved by all patients.

\section{Competing Interests}

The authors have declared that no competing interest exists.

\section{References}

1. Forouzanfar MH, Foreman KJ, Delossantos AM, Lozano R, Lopez AD, Murray CJ, et al. Breast and cervical cancer in 187 countries between 1980 and 2010: a systematic analysis. Lancet (London, England). 2011; 378: 1461-84.

2. Ojesina AI, Lichtenstein L, Freeman SS, Pedamallu CS, Imaz-Rosshandler I, Pugh TJ, et al. Landscape of genomic alterations in cervical carcinomas. Nature. 2014; 506: 371-5.

3. Wang IT, Chou SC, Lin YC. Zoledronic acid induces apoptosis and autophagy in cervical cancer cells. Tumour Biol. 2014; 35: 11913-20.

4. El Mhamdi S, Bouanene I, Mhirsi A, Bouden W, Soussi Soltani M. Cervical cancer screening: Women's knowledge, attitudes, and practices in the region of Monastir (Tunisia). Revue d'Épidémiologie et de Santé Publique. 2012; 60: 431-6.

5. Lammens T, Li J, Leone G, De Veylder L. Atypical E2Fs: new players in the E2F transcription factor family. Trends in cell biology. 2009; 19: 111-8.

6. Logan N, Graham A, Zhao X, Fisher R, Maiti B, Leone G, et al. E2F-8: an E2F family member with a similar organization of DNA-binding domains to E2F-7. Oncogene. 2005; 24: 5000 .

7. Zalmas LP, Zhao X, Graham AL, Fisher R, Reilly C, Coutts AS, et al. DNA-damage response control of E2F7 and E2F8. EMBO reports. 2008; 9: 252-9. 
8. Carvajal LA, Hamard PJ, Tonnessen C, Manfredi JJ. E2F7, a novel target, is up-regulated by p53 and mediates DNA damage-dependent transcriptional repression. Genes \& development. 2012; 26: 1533-45.

9. Westendorp B, Mokry M, Groot Koerkamp MJ, Holstege FC, Cuppen E, de Bruin A. E2F7 represses a network of oscillating cell cycle genes to control S-phase progression. Nucleic acids research. 2012; 40: 3511-23.

10. Ye L, Guo L, He Z, Wang X, Lin C, Zhang X, et al. Upregulation of E2F8 promotes cell proliferation and tumorigenicity in breast cancer by modulating G1/S phase transition. Oncotarget. 2016; 7: 23757-71.

11. Stewart CJ, McCluggage WG. Epithelial-mesenchymal transition in carcinomas of the female genital tract. Histopathology. 2013; 62: 31-43.

12. Guarino M, Rubino B, Ballabio G. The role of epithelial-mesenchymal transition in cancer pathology. Pathology. 2007; 39: 305-18.

13. Chen HZ, Tsai SY, Leone G. Emerging roles of E2Fs in cancer: an exit from cell cycle control. Nature reviews Cancer. 2009; 9: 785-97.

14. Gorgoulis VG, Zacharatos P, Mariatos G, Kotsinas A, Bouda M, Kletsas D, et al. Transcription factor E2F-1 acts as a growth-promoting factor and is associated with adverse prognosis in non-small cell lung carcinomas. The Journal of pathology. 2002; 198: 142-56.

15. Ebihara Y, Miyamoto M, Shichinohe T, Kawarada Y, Cho Y, Fukunaga A, et al. Over-expression of E2F-1 in esophageal squamous cell carcinoma correlates with tumor progression. Diseases of the esophagus : official journal of the International Society for Diseases of the Esophagus. 2004; 17: 150-4.

16. Reimer D, Sadr S, Wiedemair A, Stadlmann S, Concin N, Hofstetter G, et al. Clinical relevance of E2F family members in ovarian cancer-an evaluation in a training set of 77 patients. Clinical Cancer Research. 2007; 13: 144-51.

17. Sun J, Shi R, Zhao S, Li X, Lu S, Bu H, et al. E2F8, a direct target of miR-144, promotes papillary thyroid cancer progression via regulating cell cycle. Journal of Experimental \& Clinical Cancer Research. 2017; 36: 40.

18. Lui WO, Pourmand N, Patterson BK, Fire A. Patterns of known and novel small RNAs in human cervical cancer. Cancer Res. 2007; 67: 6031-43.

19. Parisi F, Ariyan S, Narayan D, Bacchiocchi A, Hoyt K, Cheng E, et al. Detecting copy number status and uncovering subclonal markers in heterogeneous tumor biopsies. BMC genomics. 2011; 12: 230.

20. Deng $Q$, Wang $Q$, Zong WY, Zheng DL, Wen $Y X$, Wang KS, et al. E2F8 contributes to human hepatocellular carcinoma via regulating cell proliferation. Cancer Res. 2010; 70: 782-91.

21. Lee M-Y, Shen M-R. Epithelial-mesenchymal transition in cervical carcinoma. American Journal of Translational Research. 2012; 4: 1-13.

22. Ramis-Conde I, Chaplain MA, Anderson AR, Drasdo D. Multi-scale modelling of cancer cell intravasation: the role of cadherins in metastasis. Phys Biol. 2009; 6: 016008.

23. Calaf GM, Balajee AS, Montalvo-Villagra MT, Leon M, Daniela NM, Alvarez RG, et al. Vimentin and Notch as biomarkers for breast cancer progression. Oncol Lett. 2014; 7: 721-7.

24. Martin TA, Goyal A, Watkins G, Jiang WG. Expression of the transcription factors snail, slug, and twist and their clinical significance in human breast cancer. Ann Surg Oncol. 2005; 12: 488-96.

25. Ferlay J, Soerjomataram I, Dikshit R, Eser S, Mathers C, Rebelo M, et al. Cancer incidence and mortality worldwide: sources, methods and major patterns in GLOBOCAN 2012. Int J Cancer. 2015; 136: E359-86.

26. Delgado G, Bundy B, Zaino R, Sevin B-U, Creasman WT, Major F. Prospective surgical-pathological study of disease-free interval in patients with stage IB squamous cell carcinoma of the cervix: A Gynecologic Oncology Group study. Gynecologic Oncology. 1990; 38: 352-7.

27. Jiang L, Yang Y-D, Fu L, Xu W, Liu D, Liang Q, et al. CLDN3 inhibits cancer aggressiveness via Wnt-EMT signaling and is a potential prognostic biomarker for hepatocellular carcinoma. Oncotarget. 2014; 5: 7663-76. 